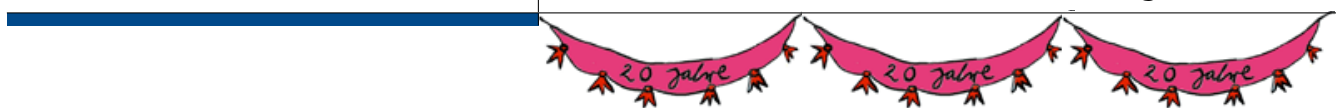

Dranginkontinenz hat sich die intravesikale intramuskuläre Applikation von Botulinumtoxin als wahre Revolution für die zum Teil extrem geplagten Patienten erwiesen und schwächt die Bedeutung der ohnehin kritischen Anwendung von oralen Anticholinergika ab.

Duloxetin zur Therapie der der weiblichen Belastungsinkontinenz wurde mit gigantischem Werberummel angekündigt, aber von den Patientinnen wegen zu geringem Effekt und mangelnder Wirkung nicht akzeptiert. Nadelsuspensionstechniken in der operativen Therapie der weiblichen Belastungsinkontinenz konnten sich ebenfalls mangels pathophysiologischer Grundlage nicht durchsetzen. Die ersten Minitapes waren wegen schlechter Verankerung mit schlechter Wirkung gefloppt. Die Nachfolgepro- dukte wurden entsprechend verbessert. Es ist für viele Entwicklungen pathognomonisch, dass ein Vorläuferprodukt oder Prototyp verrissen wird, dann aber doch verbesserte Produkte den Markt erobern.

Die Bulking Agents (Teflon, Silikon, Hyaluronsäure) zur mechanischen Abdichtung der Harnröhre als Polster zur Unterstützung des Schließapparats sind ebenfalls nach großspuriger Ankündigung schnell gescheitert. Zum Skandal avancierte die Stammzellentherapie der Belastungsinkontinenz mit der potenziellen Möglichkeit, einen neuen Muskelapparat mit pluripotenten Stammzellen zu züchten: hochrangig publiziert, dann kläglich gescheitert mit fast betrügerischer Dimension.

In der Therapie des Harnleitersteins wurden die Schlingensysteme nach
Zeiss durch eine elegante endoskopische Technik zunächst semirigide und dann standardmäßig durch flexible Techniken der Endoskopie abgelöst. War es vor 30 Jahren Standard bei Versagen der Schlinge Uretersteine offen durch eine Schnittoperation zu entfernen, so ist die Endourologie heute ein Quantensprung in der Steintherapie und natürlich auch bei der exakten Diagnostik von Tumoren. Aus der Luftfahrttechnik entwickelte sich die extrakorporale Stoßwellenlithotripsie (ESWL) von Harnsteinen ab 1982 mit großem Erfolg und ist nun wieder auf dem Rückzug bei effizienter endoskopischer Technik und aktiver Absaugung von Harnsteindesintegraten.

In der Urologie gilt letztendlich nach wie vor: KISS („Keep it simple and smart"). Dr. med. Alfons Gunnemann

\title{
Die Urologie vor 20 Jahren und heute
}

m Vergleich der Behandlung unserer Patienten damals und heute wird klar, wie ausgeprägt sich die Urologie durch viele erfolgreiche Entwicklungen, also Tops, aber auch durch die Flops, in diesen wenigen Jahren in der Praxis verändert hat.

Viele Tops in der Urologie sind im Bereich der Uroonkologie zu verzeichnen. Beim Prostatakarzinom hat sich vor allem die Indikationsstellung geändert: Wer hätte vor 20 Jahren über aktive Überwachung bei Niedrigrisikopatienten gesprochen und wer hätte bei Lymphknoten- oder Fernmetastasierung eine operative Therapie ins Auge gefasst? Die Patienten, die wir heute radikal operieren, sind völlig andere als Ende der 1990er-Jahre. Auch die Therapie selbst hat sich entscheidend entwickelt. Ein Top ist nicht nur bei der Prostata, aber hier in einer Vorreiterrolle, der Roboter. Diesen hat es in der Urologie zwar auch schon vor 20 Jahren gegeben, seinen Siegeszug hatte aber kaum jemand vorhergesehen. Auch beim kastrationsresistenten Prostatakarzinom erlangten wir große Erfolge. War das Karzinom in diesem Stadium vor zwanzig Jahren schlichtweg nicht mehr behandelbar, können wir nun auf Docetaxel, Abirateron und Enzalut- amid zurückgreifen - was für ein Paradigmenwechsel!

Als Top beim Nierenkarzinom ist $\mathrm{zu}$ werten, dass wir heute eine Nierenteilresektion bei Tumoren von 6,7 oder $8 \mathrm{~cm}$ Durchmesser durchführen und kleine Befunde völlig unbehandelt beobachten können, bei denen wir vor 20 Jahren mit Sicherheit die ganze Niere entfernt hätten. Darüber hinaus gibt es in der systemischen Therapie inzwischen eine Fülle an zugelassenen Substanzen, die das Überleben zumindest etwas verlängern. Auch beim Blasenkarzinom, wo die letzten 20 Jahre fast ohne Veränderungen vorbei gegangen zu sein schienen, zeigt sich in der systemischen Therapie mit der Einführung der Immun-Checkpoint-Inhibitoren Licht am Horizont.

Auch außerhalb der Uroonkologie sind Tops zu finden: Dabei denke ich an grundlegende Veränderungen in der Steintherapie. Die flexiblen Ureterorenoskope wurden so hervorragend weiterentwickelt, dass die Ureterorenoskopie allen anderen Methoden, auch der genialen perkutanen Mini-Nephrolitholapaxie und allemal der ESWL den Rang abgelaufenen hat. Apropos Technologie: In der Behandlung der benignen Prostatahyperplasie haben sich in den letzten 20

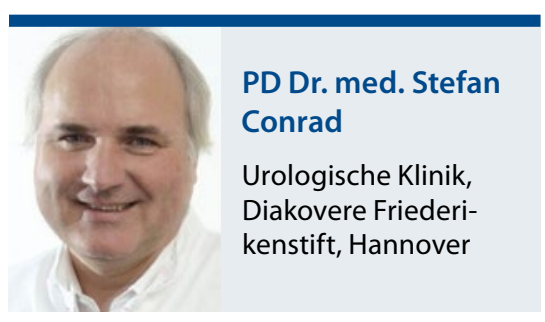

Jahren, obwohl zuvor schon jahrzehntelang eine Unzahl anderer Technologien versucht worden waren, zwei Methoden in der Laserbehandlung soweit etabliert, das sie neben der transurethralen Prostataresektion zum neuen Standard geworden sind: die Vaporisation mit dem Greenlight-Laser und die Holmium-Laser-Enukleation der Prostata.

Und was sind nun die größten Flops? Für mich sicher die PLCO-Studie, bei der durch handwerkliche Fehler ein NegativErgebnis des PSA-Screenings impliziert wurde, das weltweit das Vorsorgeverhalten einer ganzen Generation von Männern negativ beeinflusst hat. Und wohl auch der gut gemeinte, aber vorhersehbar erfolglose Versuch, mit deutscher Gründlichkeit ein für alle Mal festzulegen, was denn nun die beste Behandlungsoption beim Niedrigrisikoprostatakarzinom ist.

Wir sehen also letztendlich: Pantha rhei! Alles ist im Fluss, und wir durften in den letzten 20 Jahren mit dabei sein! PD Dr. med. Stefan Conrad 